\title{
NOTA SOBRE EL REDESCUBRIMIENTO DE TIGRIDIA HINTONII (TIGRIDIEAE: IRIDACEAE) EN EL ESTADO DE GUERRERO, MÉXICO
}

\author{
Guadalupe Munguía-Lino ${ }^{1}$, Arturo Castro-Castro ${ }^{1}$ y Aarón rodríguez ${ }^{2,3}$ \\ ${ }^{1}$ Universidad de Guadalajara, Centro Universitario de Ciencias Biológicas y \\ Agropecuarias. Apartado postal 1-139, 45110 Zapopan, Jalisco, México. \\ ${ }^{2}$ Universidad de Guadalajara, Centro Universitario de Ciencias Biológicas y \\ Agropecuarias, Departamento de Botánica y Zoología, Instituto de Botánica, \\ Herbario Luz María Villarreal, km 15.5 carretera Guadalajara-Nogales, Las Agujas, \\ Nextipac, 45110 Zapopan, Jalisco, México. \\ 33Autor para la correspondencia: rca08742@cucba.udg.mx
}

\section{RESUMEN}

Se documenta un registro nuevo de Tigridia hintonii (Iridaceae) en el estado de Guerrero, México. George B. Hinton la recolectó por primera y única vez en octubre de 1936 en Fresnos, Distrito de Mina, Zirándaro, Guerrero. Desde entonces no se habían encontrado otros ejemplares del taxon hasta el año 2012. Se incluye una descripción morfológica completa e imágenes. Además, se ofrecen datos sobre su distribución, hábitat, estado de conservación y fenología.

Palabras clave: Guerrero, México, microendemismo, Sierra Madre del Sur, Tigridieae.

\begin{abstract}
A new record of Tigridia hintonii (Iridaceae) in the state of Guerrero, Mexico, is documented. The species was first collected by George B. Hinton in Fresnos, District of Mina, Zirándaro, Guerrero in October 1936. Since then, other specimens of this taxon had not been collected until 2012. A complete morphological description and figures are provided. Additionally, data of distribution, habitat, conservation assessment and phenology are presented.
\end{abstract}

Key words: Guerrero, México, microendemism, Sierra Madre del Sur, Tigridieae. 


\section{INTRODUCCIÓN}

El género Tigridia Juss. (Tigridieae: Iridaceae) reune a plantas perennes estacionales, provistas de bulbos papiráceo-tunicados y hojas plegadas e isobilaterales. Presenta inflorescencias en forma de ripidios. Las flores se componen de tres tépalos externos y tres internos muy variables en forma, color y tamaño. Además, los tépalos internos poseen nectarios sobre la superficie adaxial que pueden estar expuestos o cubiertos por dobleces de la base de los tépalos. Una característica particular del grupo es la presencia de máculas en la base de los tépalos, parecidas a las manchas de un jaguar. Por este motivo se les nombra "oceloxochitl, flor de tigre o flor de jaguar”. Los estambres son tres y sus filamentos están fusionados formando un tubo. El estilo está partido en tres ramas, a su vez cada una se divide en dos brazos filiformes separados por un mucrón. Su fruto es una cápsula con numerosas semillas piriformes de color café rojizo. El número cromosómico básico es $\mathrm{x}=7$, con especies diploides $(2 \mathrm{n}=2 \mathrm{x}=14)$ y tetraploides $(2 \mathrm{n}=4 \mathrm{x}=28)$ (Molseed, 1970; Goldblat et al., 1998; Rodríguez y Ortiz-Catedral, 2001).

Tigridia agrupa 43 especies que se distribuyen principalmente en México y Guatemala (Munguía-Lino et al., 2015). Tigridia pavonia (L.f.) DC. es la excepción y extiende su distribución natural hasta Sudamérica, también ha sido introducida en otras partes del mundo con fines ornamentales (Vázquez, 2011). Todas las especies del género crecen en México y 35 son endémicas (Espejo-Serna, 2012; MunguíaLino et al., 2015).

El endemismo en Tigridia es marcado. Por ejemplo, T. amatlanensis Aaron Rodr. \& A. García-Mendoza, T. gracielae Aaron Rodr. \& L. Ortiz-Catedral, T. mariaetrinitatis Espejo \& López-Ferrari, T. potosina López-Ferrari \& Espejo y T. purpusii Molseed son conocidas solo de sus localidades tipo. Tigridia catarinensis Cruden, T. chrysantha Cruden \& S. J. Walker, T. estelae López-Ferrari \& Espejo, T. flammea (Lindl.) Ravenna, T. inusitata (Cruden) Ravenna, T. martinezii Calderón, T. matudae Molseed, T. mortonii Molseed, T. pugana Aaron Rodr. \& L. Ortiz-Catedral, T. rzedowskiana Aaron Rodr. \& L. Ortiz-Catedral y T. venusta Cruden solo se conocen de uno o dos municipios. Por otro lado, T. bicolor Molseed, T. chiapensis Molseed ex Cruden, T. suarezii Aaron Rodr. \& L. Ortiz-Catedral y T. tepoxtlana Ravenna son endémicas de un estado. Un caso particular es el de T. hintonii Molseed, taxon que se restringe a Guerrero, México.

La especie fue descrita con base en cinco ejemplares obtenidos por G. B. Hinton en octubre de 1936, en bosque de pino, en los alrededores de Fresnos, Distrito de Mina, municipio de Zirándaro (Fig. 1). Durante exploraciones botánicas 
recientes en la porción central de Guerrero, se recolectó material de una población en fructificación de la misma especie. Tras ser mantenidas en cultivo durante un año, las plantas florecieron y pudo ser corroborada su identidad. La descripción original de T. hintonii no incluyó datos del fruto y la de otros caracteres estaba incompleta. Por tal motivo, el objetivo del presente trabajo es dar a conocer una

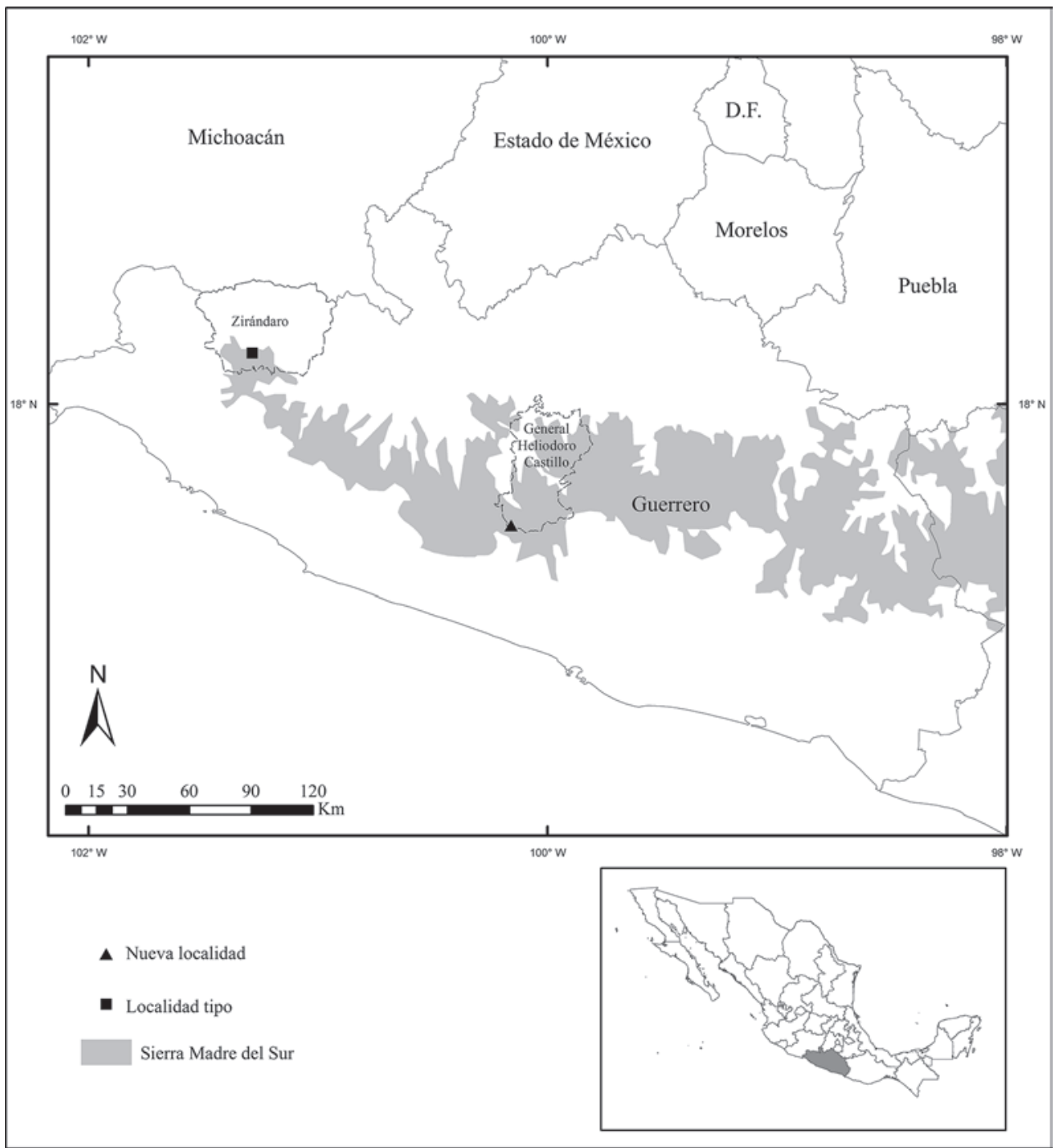

Fig. 1. Distribución geográfica de Tigridia hintonii Molseed. 
localidad nueva, así como proporcionar una descripción morfológica completa de la especie y aportar datos sobre su hábitat y estado de conservación.

\section{RESULTADOS}

Tigridia hintonii Molseed, Univ. Calif. Publ. Bot. 54:49, f. 10.1970. TIPO: MÉXICO. Guerrero, Fresnos, Distr. Mina, pine forest, 2100 m, 22.X.1936, G. B. Hinton 9748 (holotipo: UC! 1113497; isotipos: GH! 00030581, K 000322451, MICH! 1115646, US! 00092671). Fig. 2a.

Hierba perenne, 10-30 cm alto; bulbo oblongo-ovoide, 2.3-3 cm de largo, 0.9$1.2 \mathrm{~cm}$ de diámetro, pardo rojizo; tallo simple o ramificado, escabriúsculo; hojas basales y caulinares presentes, bien desarrolladas en la antesis, margen con dientes redondeados y apenas perceptibles al tacto; hoja basal solitaria, linear, 20-40 cm de largo, 0.2-0.4 cm de ancho; hojas caulinares 1-2, lineares, 22-32 cm de largo, 0.2-0.4 cm de ancho, normalmente de la misma longitud que las hojas basales, ocasionalmente sobrepasándolas; brácteas de la inflorescencia lineares, 7.5-9 cm de largo, 0.2-0.4 cm de ancho; brácteas espatáceas lanceoladas, 3-4 cm de largo, 0.3-0.5 cm de ancho; flores (1-)2 por ripidio, 2-3 cm de diámetro, tépalos formando una región crateriforme en la base, blancos con máculas de color púrpura en la base (Fig. 3a); tépalos exteriores extendidos, con los ápices reflejos, ampliamente ovados, 2-2.5 cm de largo, 1-1.5 cm de ancho (Fig. 3b); tépalos internos unguiculados, hastadocordados, $1.5 \mathrm{~cm}$ de largo, $1 \mathrm{~cm}$ de ancho, ápice agudo, con nectarios convolutos, transparentes (Fig. 3c flecha negra); filamentos fusionados hasta cerca del ápice, columna 6-8 mm de largo; anteras 5-7 mm de largo; ramas del estilo falcadas entre las anteras, bífidas, 4-5 mm de largo, con un mucrón de 1-2 mm de largo en el seno (Fig. 3d-e flecha negra); cápsulas maduras oblongo-ovoides, 1.2-1.8 cm de largo, 0.4-0.6 cm de diámetro, el ápice purpúreo; semillas ovado-piriformes, $0.2 \mathrm{~mm}$ de largo, 0.1-0.2 mm de diámetro, de color pardo.

Distribución y hábitat. Tigridia hintonii es endémica de Guerrero. Se conoce de dos sitios en la Sierra Madre del Sur. El nuevo material recolectado documenta su presencia en el municipio de General Heliodoro Castillo (Fig. 1). Habita sobre taludes rocosos dentro del bosque de pino y el bosque de pino-encino-abeto, entre los 2100 y $2926 \mathrm{~m}$ de elevación. Las especies asociadas más frecuentes son Abies guatemalensis Rehder, Dahlia coccinea Cav., Dahlia hintonii Sherff, Echeandia spp., Calceolaria mexicana 
Munguía-Lino et al.: Redescubrimiento de Tigridia hintonii en Guerrero, México

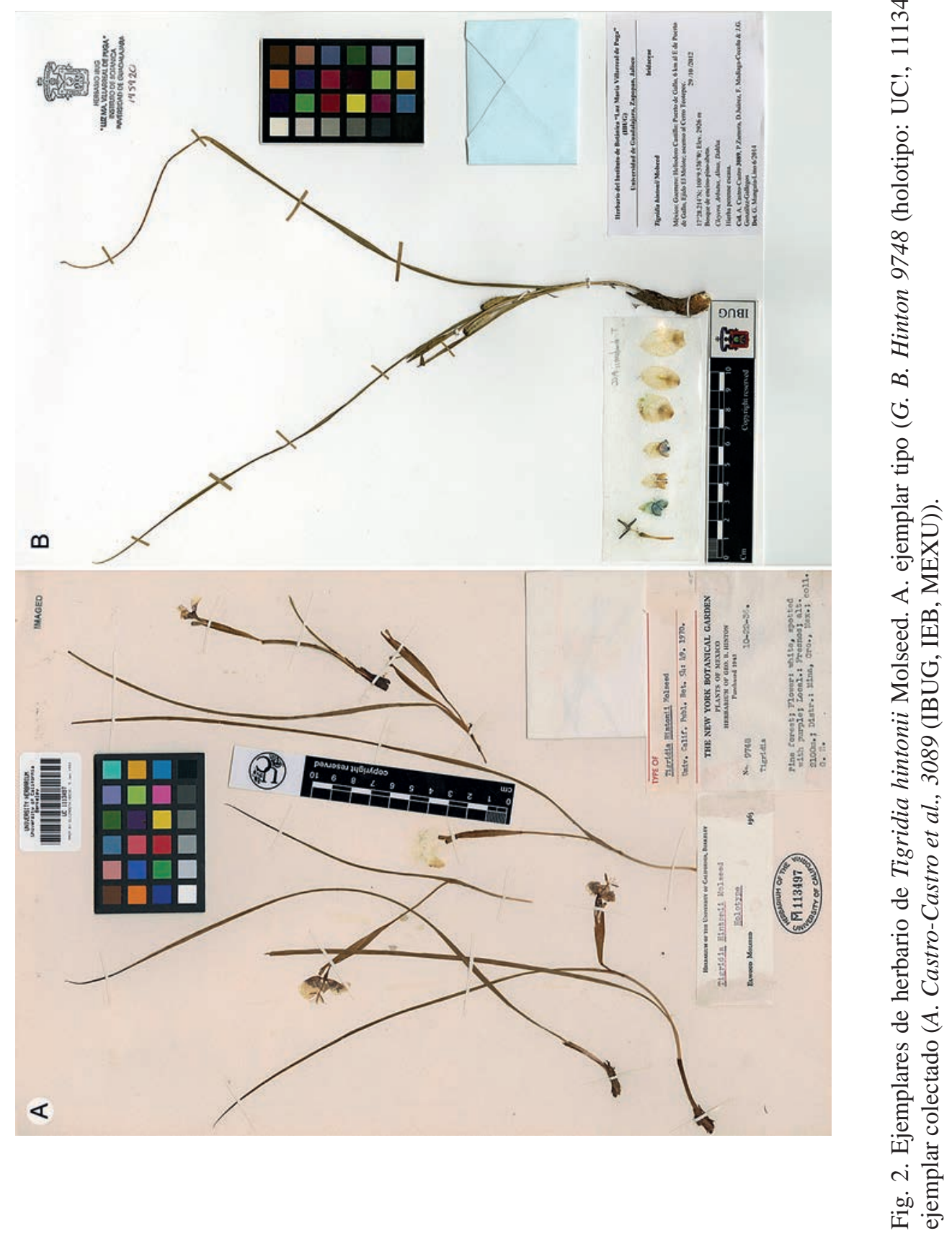



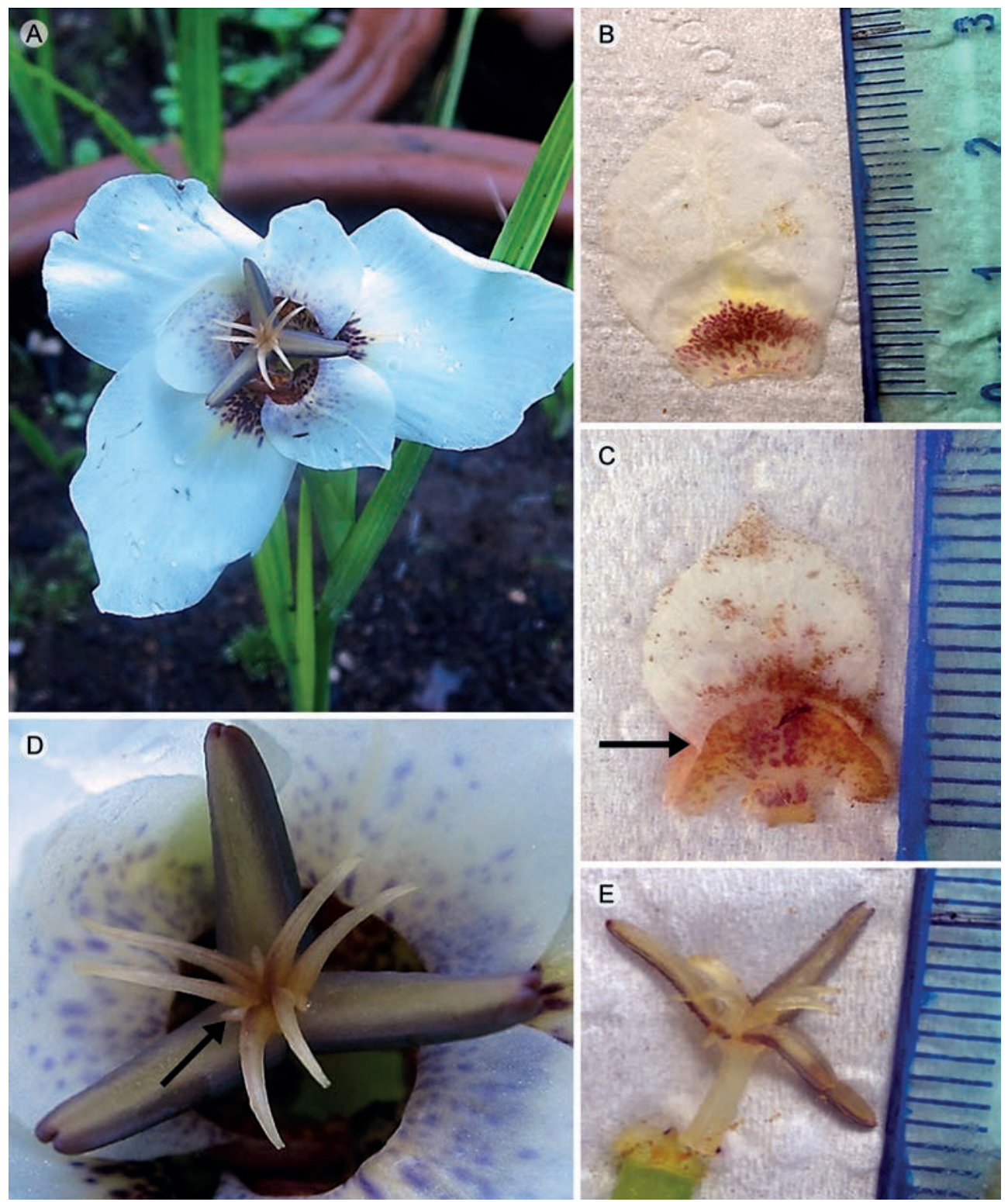

Fig. 3. Morfología floral de Tigridia hintonii Molseed. A. flor, con los tépalos formando una pequeña región crateriforme en la base; B. tépalo externo; C. tépalo interno con nectarios convolutos (flecha); D-E. vista frontal y lateral del gineceo y androceo. Fotografías de L. M. Vázquez García, a partir de ejemplares cultivados (A. Castro-Castro et al. 3089 (IBUG, IEB, MEXU)). 
Benth., Clethra sp., Manfreda spp., Pinus chiapensis (Martínez) Andresen, Quercus spp., Salvia spp., Sedum oxypetalum Kunth, Solanum iopetalum (Bitter) Hawkes, Stevia spp., Tigridia inusitata (Cruden) Ravenna, Utricularia sp. y Valeriana spp.

Estado de conservación. Actualmente Tigridia hintonii se conoce de dos poblaciones disyuntas y separadas entre sí por $150 \mathrm{~km}$ en línea recta y tiene un área de ocupación de $8 \mathrm{~km}^{2}$. Con estas características se puede definir el estado de conservación mediante los criterios y categorías de la Lista Roja de la Unión Internacional para la Conservación de la Naturaleza (UICN; Anónimo, 2012). De acuerdo con el criterio distribución geográfica (B) representada por el área de ocupación (AOO, B2(a)), en la categoría Peligro Crítico de Extinción se encuentran los taxa que tengan AOO menores de 10 km² (Bachman et al., 2011; Anónimo, 2012). Debido a lo anterior es posible ubicar a T. hintonii como una especie en Peligro Crítico de extinción (CR, criterio B2(a)). Sin embargo, es necesario evaluar los criterios A, C, D y E para su posterior inclusión en la Lista Roja de la UICN.

Fenología. Tigridia hintonii florece y fructifica de agosto a octubre. En condiciones de cultivo floreció en julio.

Especímenes examinados. MÉXICO. Guerrero. Municipio de General Heliodoro Castillo, $6 \mathrm{~km}$ al E de Puerto del Gallo rumbo a Filo de Caballo, Ejido el Molote, ascenso al Cerro Teotepec, alt. 2926 m, 17²8'2.84" N, 1009'34.56" W, 29.X.2012, A. Castro-Castro et al. 3089 (IBUG, IEB, MEXU). Fig. 2b.

\section{DISCUSIÓN}

Las plantas de Tigridia hintonii miden entre 10 y $30 \mathrm{~cm}$ de alto, aunque en el campo aparentan una talla menor al estar cubiertas por musgos que ocultan hasta la mitad de su tamaño. Se caracterizan por la presencia de las hojas basales y caulinares durante la antesis, los tépalos blancos con manchas de color púrpura en el centro y los internos muy reducidos en la parte distal (Figs. 1 y 2). Es probable que en el ejemplar tipo la flor no había sido medida y observada con detalle. Molseed (1970) describió la especie a partir de especímenes en los cuales la flor no está debidamente preservada. En la descripción menciona que el ápice de los tépalos internos está completamente abortado, quedando el limbo reducido a un mucrón. En este estudio se midió este carácter en material fresco y a partir de disecciones 
de la flor en fresco y luego deshidratadas. A diferencia de lo propuesto por Molseed (1970), los tépalos interiores de T. hintonii son unguiculados, hastado-cordados, de $1.5 \mathrm{~cm}$ de largo y de $1 \mathrm{~cm}$ de ancho con el ápice agudo (Fig. 3c). Asimismo, el fruto y las semillas no fueron incluidos en la descripción original, ya que el ejemplar tipo no los presenta.

Debido a las características de sus nectarios, es posible ubicar a Tigridia hintonii en el subgénero Tigridia propuesto por Molseed (1970). Esta especie es morfológicamente similar a T. mexicana ssp. passiflora Molseed (Fig. 4b), la cual presenta tépalos blancos con manchas pardas en la base. La diferencia entre ambos taxones es la presencia de hojas durante la antesis y la reducción de la parte distal de los tépalos internos en T. hintonii. Molseed (1970) relacionó a T. hintonii
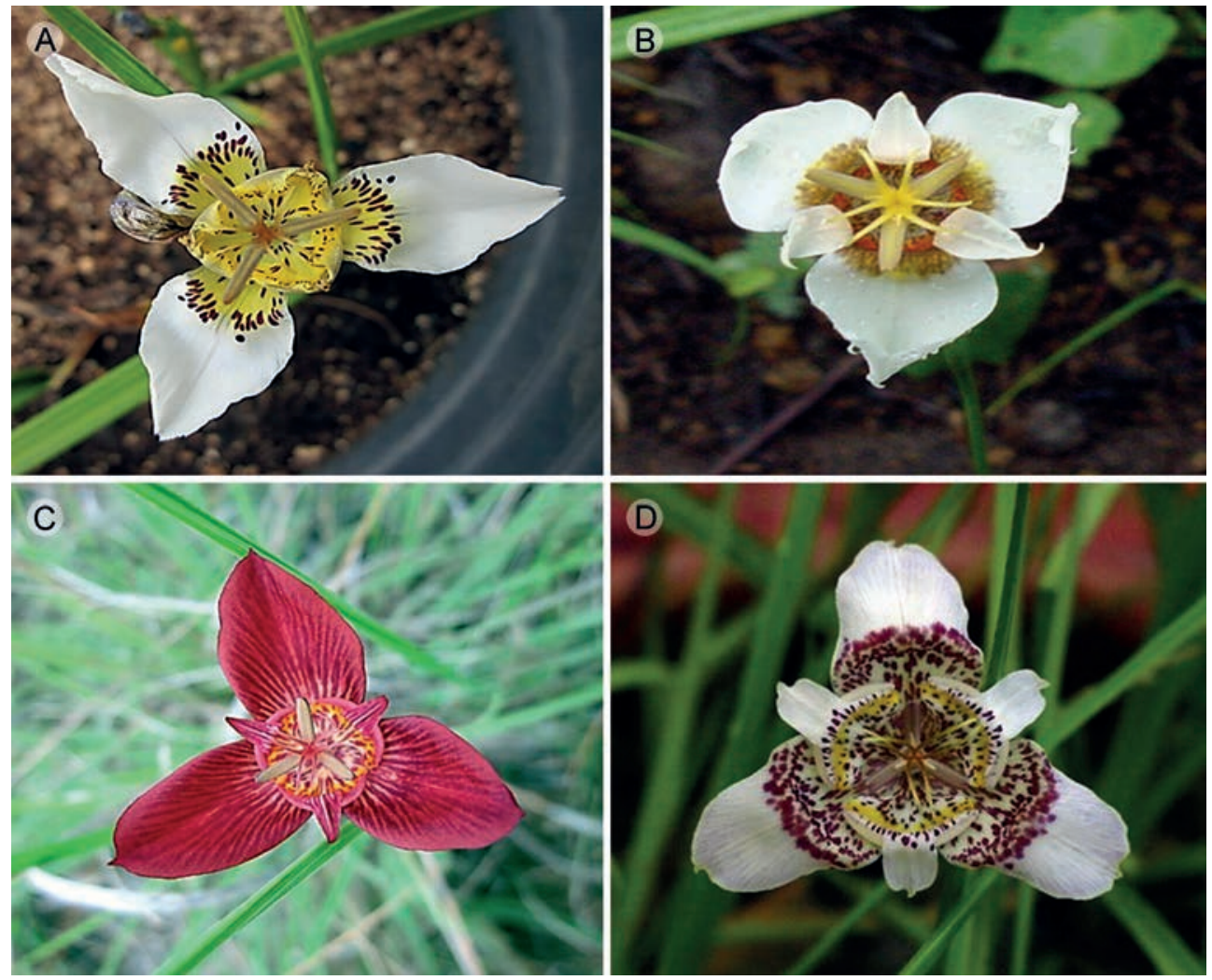

Fig. 4. Flores de especies relacionadas a Tigridia hintonii. A. T. chiapensis; B. T. mexicana ssp. passiflora; C. T. mortonii; D. T. tepoxtlana. Fotografías de G. Munguía Lino (A y C) y A. Rodríguez Contreras (B y D). 
Munguía-Lino et al.: Redescubrimiento de Tigridia hintonii en Guerrero, México

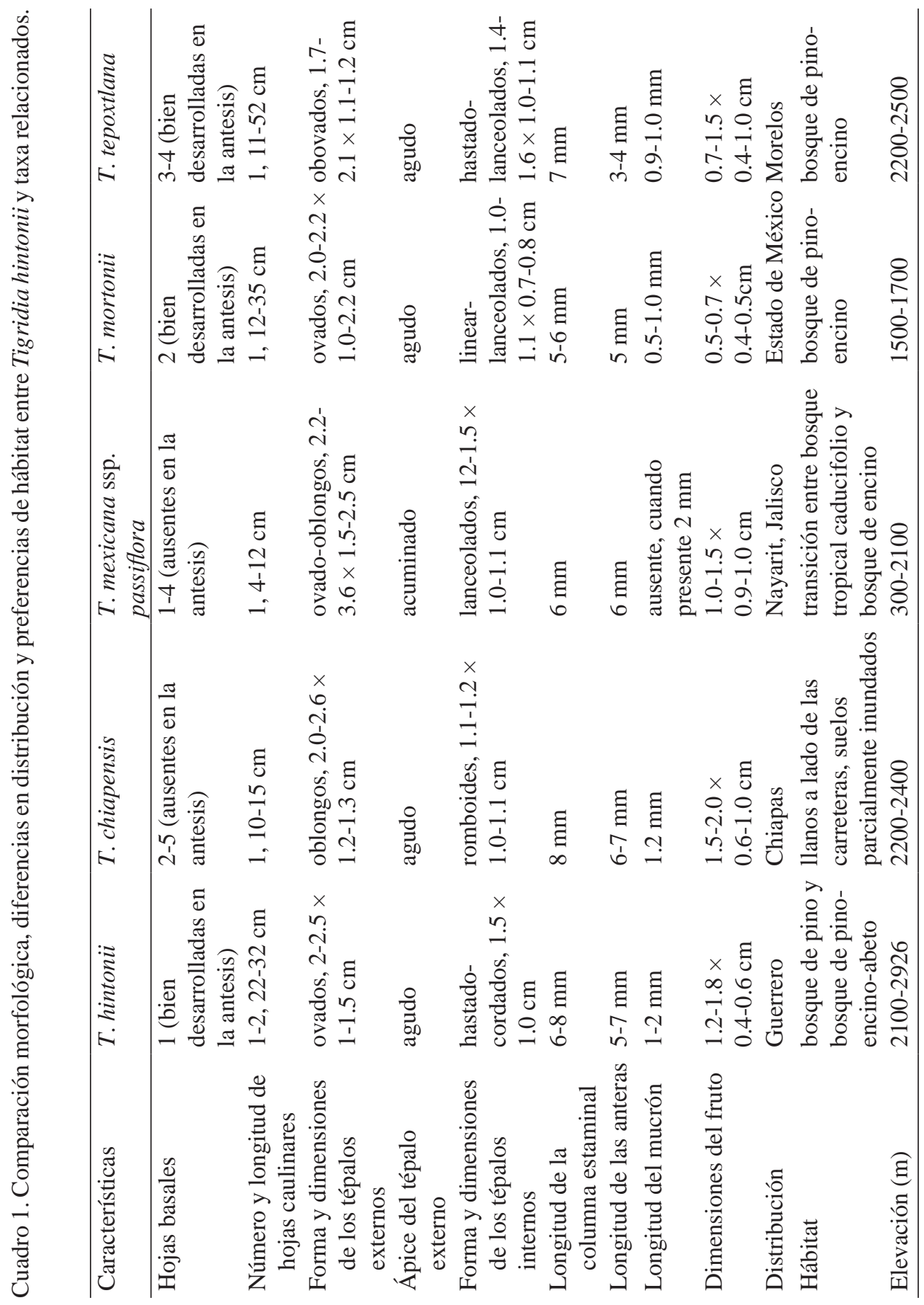


con T. chiapensis Molseed ex Cruden (Fig. 4a), T. mortonii Molseed (Fig. 4c) y $T$. tepoxtlana Ravenna (Fig. 4d). Las diferencias y similitudes entre ellas se muestran en el Cuadro 1.

Cabe señalar que el taxon está incluido en la lista de especies en riesgo de la norma oficial mexicana NOM-059-SEMARNAT-2010, bajo la categoría de protección especial (Anónimo, 2010), pero no se encuentra en la lista roja de la UICN (Anónimo, 2012), por lo que requiere ser evaluado. Al igual que otras especies del género, $T$. hintonii podría aprovecharse como ornamental. Un programa de manejo adecuado de la especie repercutiría en beneficio de las poblaciones de la planta y de las comunidades rurales con recursos limitados.

\section{AGRADECIMIENTOS}

Agradecemos a Luis Miguel Vázquez García por las fotografías proporcionadas. Nuestra gratitud para Bladimira Nepomuceno Cantú, Pilar Zamora Tavares, Roselia Sánchez Reyna, Sulibeth Nava Rizo, Adalberto Navarrete Arreola, Alfonso Mariano Emigdio, Daniel Cervantes Román, Diego Benito Basulto, Francisco Salvador Maradiaga Ceceña y Jesús González Gallegos por su apoyo durante el trabajo de campo. Se agradece al Consejo Nacional de Ciencia y Tecnología (CONACYT), por la beca otorgada para realizar los estudios de posgrado a GML (251844) y ACC (229039, 335340), y a la fundación Idea Wild por el equipamiento otorgado. Por último, gracias al personal de los herbarios CHAP, CHAPA, CHIP, CIIDIR, CIMI, CREG, ENCB, FCME, FEZA, GH, GUADA, HUMO, IBUG, IEB, K, MEXU, MICH, OAX, QMEX, SERO, UAGC, UC, UAMIZ, XAL, XALU y ZEA por las facilidades cedidas durante la revisión de ejemplares.

\section{LITERATURA CITADA}

Anónimo. 2010. Norma Oficial Mexicana NOM-059-ECOL-2010, Protección ambientalespecies nativas de México de flora y fauna silvestres-Categorías de riesgo y especificaciones para su inclusión, exclusión o cambio-Lista de especies en riesgo. Diario Oficial de la Federación. 30 de diciembre de 2010. Segunda Sección. México.

Anónimo. 2012. IUCN red list categories and criteria. Versión 3.1. IUCN Species Survival Commission. IUCN. Gland, Suiza y Cambridge, UK. <http://jr.iucnredlist.org/ documents/redlist_cats_crit_en.pdf.> Consultado en septiembre 2014. 
Bachman, S., J. Moat, A. W. Hill, J. de la Torre y B. Scott. 2011. Supporting red list threat assessments with GeoCAT: geospatial conservation assessment tool. Zookeys 150: $117-126$.

Espejo-Serna, A. 2012. El endemismo de las Liliopsida mexicanas. Acta Bot. Mex. 100: 195-257.

Goldblat, P., J. C. Manning y P. Rudall. 1998. Iridaceae. In: Kubitzki, K. (ed.). The families and genera of vascular plants III: flowering plants, monocotyledons, Lilianae (except Orchidaceae). Springer. Berlín, Alemania. pp. 295-335.

Molseed, E. 1970. The genus Tigridia (Iridaceae) of Mexico and Central America. Univ. Calif. Publ. Bot. 54: 1-113.

Munguía-Lino, G., G. Vargas-Amado, L. M. Vázquez-García y A. Rodríguez. 2015. Riqueza de especies y distribución geográfica de la tribu Tigridieae (Iridaceae) en Norteamérica. Rev. Mex. Biodiv. 86: 80-98.

Rodríguez, A. y L. Ortiz-Catedral. 2001. La tribu Tigridieae (Iridaceae) en México. ScientiaCUCBA 32: 123-136.

Vázquez, G. L. M. 2011. Tigridias ornamentales, uso y distribución. Universidad Autónoma Chapingo. Texcoco, México. 108 pp. 